\title{
LDB2 regulates the expression of DLL4 through the formation of oligomeric complexes in endothelial cells
}

\author{
Hyun-Jung Choi ${ }^{1, *}$, Seung-Sik Rho ${ }^{2}$, Dong-Hoon Choi ${ }^{2}$ E Young-Guen Kwon ${ }^{2, *}$ \\ ${ }^{1}$ Severance Integrative Research Institute for Cerebral \& Cardiovascular Diseases (SIRIC), College of Medicine, Yonsei University, Seoul \\ 03722, ${ }^{2}$ Department of Biochemistry, College of Life Science and Biotechnology, Yonsei University, Seoul 03722, Korea
}

Delta-like ligand 4 (DLL4) expression in endothelial cells is intimately associated with angiogenic sprouting and vascular remodeling, but the precise mechanism of transcriptional regulation of DLL4 remains incompletely understood. Here, we showed that LIM-domain binding protein 2 (LDB2) plays an important role in regulating basal DLL4 and VEGF-induced DLL4 expression. Knockdown of LDB2 using siRNA enhanced endothelial sprouting and tubular network formation in vitro. Injection of $\boldsymbol{I d b 2}$-morpholino resulted in defective development of intersegmental vessels in zebrafish. Reduction or overexpression of LDB2 in endothelial cells decreased or increased DLL4 expression. LDB2 regulated DLL4 promoter activity by binding to its promoter region and the same promoter region was occupied and regulated by the LMO2/TAL1/GATA2 complex. Interestingly, LDB2 also mediated VEGF-induced DLL4 expression in endothelial cells. The regulation of DLL4 by the LDB2 complex provides a novel mechanism of DLL4 transcriptional control that may be exploited to develop therapeutics for aberrant vascular remodeling. [BMB Reports 2018; 51(1): 21-26]

\section{INTRODUCTION}

Well-coordinated sprouting angiogenesis is essential for formation of functional vascular networks during development and adulthood. DLL4 plays a critical role in endothelial sprouting and vascular remodeling relevant in physiologic and pathologic angiogenesis $(1,2)$. DLL4 expression is mainly detected in the restricted region of nascent vessels, particularly

*Corresponding authors. Young-Guen Kwon, Tel: +82-2-2123-5697; Fax: +82-2-362-9897; E-mail: ygkwon@yonsei.ac.kr; Hyun-Jung Choi, Tel: +82-2-2228-0927; Fax: +82-2-2227-7906; E-mail: hjchoi12@ yuhs.ac

https://doi.org/10.5483/BMBRep.2018.51.1.140

Received 21 July 2017, Revised 14 August 2017, Accepted 14 September 2017

Keywords: Angiogenesis, DLL4, Endothelial cells, LDB2, LMO2/ TAL1/GATA2 complex, Sprouting in tip cells, which is associated with VEGF signaling. VEGF induces the tip cell phenotype and DLL4 expression $(3,4)$ and triggers Notch signaling in adjacent cells, and this, in turn, attenuates VEGF signaling and inhibits tip cell behavior $(5,6)$, thereby balancing vessel sprouting. Gene inactivation or pharmacological inhibition of DLL4 or Notch results in excessive sprouting with an increased number of tip cells at the vascular front and hyperdense vascular networks $(3,4,6)$. Despite the central role of DLL4 in vascular remodeling and angiogenesis, the precise transcriptional regulatory mechanism of DLL4 is less well defined.

LIM-domain binding proteins (LDBs) were initially identified as co-factors for LIM-homeodomain (LHX) transcription factors and LIM-only (LMO) proteins (7), which play critical roles in cell fate determination, differentiation, and tissue development $(8,9)$. LDBs were also shown to interact with other transcription factors, including GATA, bHLH, and Otx family members $(10,11)$. The LDB family is composed of two members, LDB1 and LDB2, which are highly homologous in two well conserved domains: an amino-terminal homodimerization domain (DD) and a carboxyl-terminal LIM interaction domain (LID) (9). These domains play important roles in mediating transcription through higher-order transcription complexes containing TAL1/E47/GATA1/LMO2/LDB. Of particular interest is that LDB1 mediates long-range promoter-enhancer interactions through these multimeric complexes, which regulate erythroid gene transcription $(11,12)$. Although the roles of LDB1 in diverse developmental processes, including erythroid differentiation, are relatively well established, the function of LDB2 has not yet been clearly determined. In this study, we determined that LDB2 plays a key role in angiogenic sprouting by regulating DLL4 through higher-order transcription complexes in endothelial cells.

\section{RESULTS}

Depletion of LDB2 in endothelial cells leads to aberrant tubular network formation and endothelial sprouting To identify transcription factors that are critical for the angiogenic activity of endothelial cells, we screened transcription factors whose expressions were significantly altered during endothelial progenitor cell (EPC) differentiation from a

ISSN: 1976-670X (electronic edition)

Copyright (C) 2018 by the The Korean Society for Biochemistry and Molecular Biology

c) This is an open-access article distributed under the terms of the Creative Commons Attribution Non-Commercial License (http://creativecommons.org/licenses/by-nc/4.0) which permits unrestricted non-commercial use, distribution, and reproduction in any medium, provided the original work is properly cited. 
microarray analysis (GEO accession number GSE12891). The mRNA of LDB2 was dramatically induced during EPC differentiation, whereas mRNA expression of LDB1, a homologue, was not significantly changed (Supplementary Fig. 1). To address the role of LDB2 in endothelial cells, we performed tubular network formation assay on Matrigel with endothelial cells transfected with siRNA targeting LDB2 (Supplementary Fig. 2A-C). Number of meshes formed on Matrigel increased in LDB2-deficient endothelial cells when compared to control cells (Supplementary Fig. 2B and C) A 3D-fibrin-gel bead assay with LDB2-deficient endothelial cells displayed more sprouting, and the length of sprouts were increased compared to control cells (Fig. 1A-C). To investigate the role of $1 \mathrm{db} 2$ during angiogenesis in vivo, we knock-downed $l d b 2$ gene expression using morpholino and observed intersegmental trunk vessels (ISV) in transgenic zebrafish embryos with fluorescent blood vessels (refered to as $\operatorname{Tg}(f / k-1$ :egfp) embryos). At $72 \mathrm{hpf}$, control MO-injected embryos showed no apparent vascular abnormality (Fig. 1D and F, Control MO). By contrast, ldb2

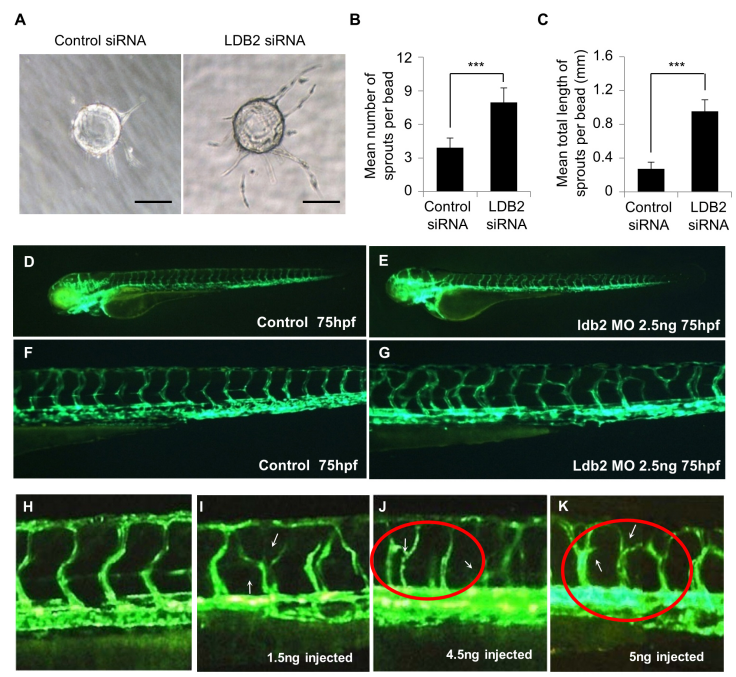

Fig. 1. Depletion of LDB2 in endothelial cells results in abnormal endothelial sprouting. (A-C) HUVECs were transfected with control or LDB2 siRNA (80 $\mathrm{nM}$ ) and 3-D fibrin gel assay was performed. (A) Representative images of sprouting from HUVECs transfected with indicating siRNA (80 nM). Sacale bars: $100 \mu \mathrm{m}$ (B and C) Quantification of sprouts in a 3-D-fibrin gel assay. Beads coated with HUVECs were seeded and cultured in fibrin gel for 5 days. ( $n=4 ; 15$ beads were assayed in each experiment). (D-G) Control morpholino (D and F) or Idb2 morpholino ( $E$ and $G$ ) was microinjected at one cell stage of zebrafish and pictures were taken at $75 \mathrm{hpf}$ to examine the vessel development. High magnification of control embryo's trunk region (F) showing no abnormality and Idb2 morpholino-injected embryo's trunk region (G) showing aberrant development of intersegmental vesseles. (H-K) Control or ldb2 morpholino of $1.5 \mathrm{ng}, 4.5 \mathrm{ng}$ and $5 \mathrm{ng}$ was injected. $/ \mathrm{db} 2$ morpholino-injected embryos show disorganized and hyper-branched intersegmental vessels (I-K, red rounds and arrows). Error bars represent mean $\pm \mathrm{SD}$. ${ }^{* * * P}<0.001$. Student's $t$ test.
MO-injected Tg(flk-1:egfp) embryos displayed vessel-branching defects (Fig. $1 \mathrm{E}$ and $\mathrm{G}, \quad l d b 2 \mathrm{MO}$ ). High-magnification images confirmed that ISVs of $I d b 2$ MO-injected embryo was disorganized and hyper-branched at this stage in a dose- dependent manner (Fig. $1 \mathrm{H}-\mathrm{K}$ ).

\section{LDB2 regulates transcription of DLL4 in endothelial cells}

The increased sprouting and hyper-dense network formation by LDB2-knockdowned endothelial cells is reminiscent of the behavior of DLL4-deficient endothelial cells (13). To elucidate the relationship between DLL4 and LDB2 in endothelial cells, DLL4 protein levels were measured in LDB2-deficient endothelial cells. The level of DLL4 protein expression was significantly reduced (Fig. 2A). Because LDB2 is a transcription co-factor, we examined transcription of DLL4 after LDB2 knockdown in HUVECs and found that the transcript level and promoter activity of DLL4 decreased (Fig. 2B and C). Conversely, DLL4 expression was increased when LDB2 was overexpressed in HUVECs (Fig. 2D). DLL4 promoter activity was also increased with LDB2 expression in a dose-dependent manner, although the induction level was relatively low (Fig. 2E). This moderate change may be attributed to the fact that LDB2 is a transcription co-factor, which requires other transcription factors for full induction of transcription of a target promoter.
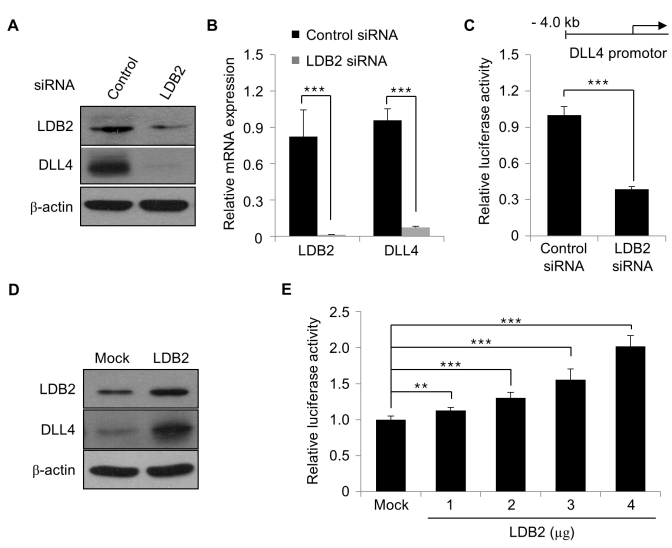

Fig. 2. LDB2 regulates transcription of DLL4 in endothelial cells. (A, B) HUVECs were transfected with control or LDB2 siRNA (80 $\mathrm{nM})$ and DLL4 protein and transcript level was determined by Western blot analysis (A) and RT-PCR (B). (C) Luciferase activity in cells co-transfected with LDB2 siRNA and luciferase reporter containing human DLL4 promoter $(-4.0 \mathrm{~kb})$. (D) HUVECs were transfected pCMV2-FLAG mock $(1 \mu \mathrm{g})$ or pCMV2-FLAG-LDB2 vector $(1 \mu \mathrm{g})$ and DLL4 proteins level was determined by Western blot analysis. (E) HUVECs were transfected with the DLL4 promoter- luciferase reporter vector along with increased amount of LDB2- expression vector. Luciferase activity was measured. Each experiment was repeated at least 3 times. Error bars represent mean \pm standard deviation (SD). $* * P<0.01 ; * * * P<$ 0.001 . Student's $t$ test. 
LDB2 regulates DLL4 transcriptionally through the multimeric complex consisting of LMO2, TAL1, and GATA2 onto DLL4 promoter

LDB2 regulates genes by interacting with transcriptional factors that directly bind to DNA, and several transcription factors have been identified as binding partners of LDB proteins. LDB proteins are known to bind TAL1/GATA transcription factors, and this interaction is mediated by LMO2. Interestingly, TAL1/GATA binding sites were found $-1.9 \mathrm{~kb}$ from the DLL4 promoter region, and this organization is highly conserved in mice and humans (Fig. 3A and Supplementary Fig. 3). Activation of the mouse DLL4 promoter
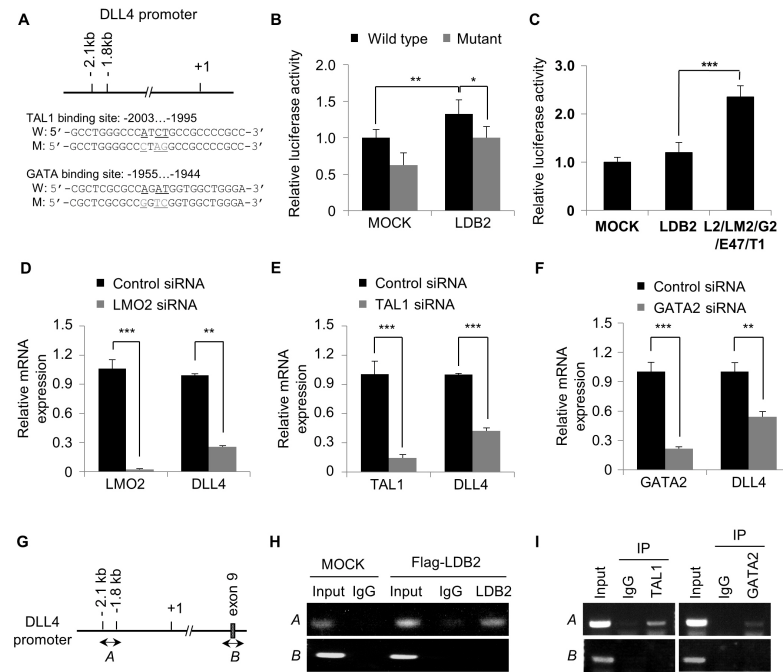

Fig. 3. LDB2 regulates DLL4 promoter activity via TAL1 and GATA2 binding on DLL4 promoter. (A) The human DLL4 promoter region containing the E-box-GATA2 element (binding sites for TAL1 and GATA2) is depicted. Wild-type (W) and mutant (M) sequences are shown. The point mutations are underlined. (B) HUVECs were transfected with wild-type or mutant DLL4 promoters fused to a luciferase reporter along with a pCMV2-FLAG or pCMV2-FLAG-LDB2 expression vector. Luciferase activity was measured $24 \mathrm{~h}$ after transfection. (C) HUVECs were transfected with a mock vector, an LDB2 vector, or an LDB2 (L2), LMO2 (LM2), GATA2 (G2), E47, and TAL1 (T1) vector along with a DLL4 promoter-luciferase reporter. The total amount of transfected DNA was adjusted to $4 \mu \mathrm{g}$. (D-F) DLL4 transcripts were measured in HUVECs transfected with indicated siRNA by qRT-PCR. Error bars represent mean \pm SD. $* * \mathrm{P}<0.01, * * * \mathrm{P}<0.001$ by Student's $t$ test. (G) Primers corresponding to the region that encompasses the E-box-GATA2 element of the DLL4 promoter are denoted as A and primers corresponding to the DLL4 coding region are denoted as $\mathrm{B}$ for CHIP analysis. (H) HUVECs were transfected with the pCMV2-FLAG mock vector or the pCMV2-FLAG-LDB2 vector, and FLAG-LDB2 was immunoprecipitated with an anti-FLAG antibody. Co-immunoprecipitated and input DNAs were amplified with the primers indicated in G. (I) Endogenous TAL1 and GATA2 were immunoprecipitated in HUVECs with anti-TAL1 and anti-GATA2 antibodies, respectively. Co-immunoprecipitated and input DNAs were amplified with primers indicated in G. Each experiment was repeated at least 3 times. by LDB2 protein was suppressed when the promoter region containing TAL1/GATA binding sites was deleted (Supplementary Fig. 3). Site-directed mutagenesis of these sites also suppressed LDB2-induced and basal DLL4 promoter activity (Fig. 3B).

To determine whether this transcription factor complex regulates transcription from the DLL4 promoter, the components of the complex were overexpressed in HUVECs along with the DLL4 promoter fused to luciferases. The promoter activity of DLL4 was significantly enhanced by cotransfection with TAL1, E47, GATA2, LMO2, and LDB2 (Fig. 3C). In accordance with this, DLL4 transcription was reduced when TAL1, GATA2, or LMO2 was knocked-down by siRNA in HUVECs (Fig. 3D-F). These results suggest that the TAL1/E47/GATA2/LMO2 complex bound by LDB2 may play a significant role in transcriptional regulation of DLL4 in endothelial cells. To further elucidate the transcriptional regulation of DLL4 by LDB2, we performed chromatin immunoprecipitation in LDB2-overexpressing HUVECs. LDB2-FLAG was immunoprecipitated at the TAL1 and GATA2 binding sites of the DLL4 promoter (Fig. 3G and $\mathrm{H}$ ). Endogenous TAL1 and GATA2 were also detected at the same promoter region (Fig. 3I).

\section{LDB2 mediates VEGF-induced DLL4 expression}

It is well known that VEGF induces DLL4 transcriptionally in endothelial cells, and thereby restricts tip cell formation in adjacent cells. To determine whether DLL4 induction by VEGF is regulated by LDB2 in endothelial cells, HUVECs transfected with a dominant negative form of LDB2 (LDB2 DN; the

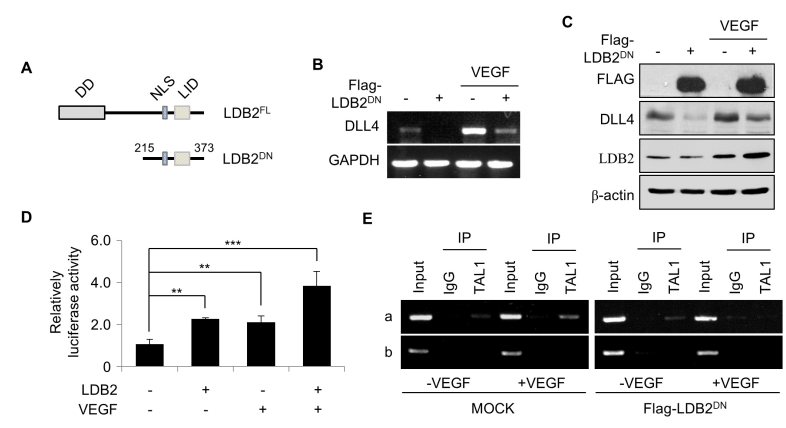

Fig. 4. LDB2 mediates VEGF-induced DLL4 expression. (A) The dominant negative form of LDB2 (LDB2 ${ }^{\mathrm{DN}}$, a.a. 215-373) was depicted. $\mathrm{LDB}_{2}^{\mathrm{FL}}$, full length form of LDB2; NLS, nuclear localization sequence; LID, LIM-interacting domain. ( $\mathrm{B}$ and $\mathrm{C}$ ) HUVECs were transfected with pCMV2-FLAG or pCMV2-FLAG-LDB2 ${ }^{\mathrm{DN}}$ and treated with VEGF $(50 \mathrm{ng} / \mathrm{ml})$. (B) mRNA of DLL4 was analyzed by RT-PCR. (C) Expression of DLL4 and LDB2 protein was detected by western blotting. (D) HUVECs transfected with mock vector or with LDB2 vector and with the DLL4 promoter-luciferase reporter were treated with VEGF. Luciferase activity was determined. (E) CHIP assays were performed with lysates from HUVECs transfected with mock vector or with the LDB2 ${ }^{\mathrm{DN}}$ expression vector. Endogenous TAL1 was immunoprecipitated $1 \mathrm{~h}$ after VEGF treatment. Each experiment was repeated at least 3 times. Error bars represent mean $\pm \mathrm{SD}$. ${ }^{* * P}<0.01,{ }^{*} * \mathrm{P}<0.001$ by Student's $t$ test. 
dimerization-domain deletion mutant) (Fig. 4A). Transcription of DLL4 was induced by VEGF in control cells, and this induction of DLL4 mRNA by VEGF was suppressed in LDB2 DN-expressing cells (Fig. 4B). Expression of DLL4 protein was also induced by VEGF. Similar to DLL4 expression, endogenous LDB2 protein was increased by VEGF treatment. However, the induction of expression of DLL4 protein by VEGF was inhibited by LDB2 DN (Fig. 4C). The reporter activity of the DLL4 promoter was increased by VEGF treatment, and this was further enhanced by LDB2 expression (Fig. 4D). Moreover, chromatin immunoprecipitation results revealed that binding of TAL1 to the DLL4 promoter region was also increased by VEGF treatment and this increase was suppressed by expression of LDB2 DN (Fig. 4E). These results suggest that LDB2 is involved in VEGF induction of DLL4 through its oligomerization.

\section{DISCUSSION}

LDB transcription co-factor proteins have been suggested to be important for the regulation of cell selection and cell fate determination via their interactions with LIM domain containing transcription factors $(7,14)$. In this study, we showed that LDB2 is expressed in endothelial cells and that it plays a unique role in vascular remodeling by regulating expression of DLL4. Despite the incomparable role of DLL4 expression on vascular morphogenesis, transcriptional regulation of DLL4 in endothelial cells has remained largely unclear. A few transcription factors, including Wnt signaling effector, $\beta$-catenin, and FOXC transcription factors that regulate DLL4 expression in endothelial cells have been identified (15-17), but these studies did not provide direct evidence of their roles in VEGF-induced DLL4 expression. The current study provides a new insight into the transcriptional regulation of DLL4 expression and shows that an LDB2-containing higher-order transcriptional complex mediates basal and VEGF-induced DLL4 expression. ETS transcription factors have also been identified as regulators of DLL4 expression and they primarily function during arterial specification (18). SOXF transcription factors and the RBPJ/NICD complex also regulate DLL4 transcription during arterial specification (19). Because these studies focused on regulation of arterial-specific DLL4 expression during development, they did not evaluate regulation of DLL4 expression during endothelial sprouting. Further, the regulatory elements bound by these factors are in intron 3 of DLL4 rather than in the promoter sites identified in this study. Thus, it is likely that the mechanisms regulating DLL4 expression during arterial specification and during angiogenic sprouting are distinct.

TAL1, a transcription factor initially identified in human acute T-cell leukemia, has been shown to be involved in both developmental and postnatal angiogenesis $(20,21)$. TAL1 deletion mutant mice showed defective yolk sac angiogenesis due to the defect in Tal1-/- endothelial cells. TAL knockdown in HUVECs resulted in defective cell migration, which led to a lack of tubular network formation on Matrigel (22). VEcadherin has been identified as a target of the TAL1 complex in endothelial cells (23). Deletion of LMO2, which interacts with LDB2 and TAL1/GATA2, also caused a severe angiogenesis defect in mouse embryos (24). The vascular defects caused by these genetic deficiencies were not due to impaired formation of the primitive vascular plexus, but rather were due to defects in subsequent angiogenic remodeling processes. The results from these studies and our study suggest that misregulated expression of DLL4 by the lack of these transcription factors may be the cause of the abnormal vascular remodeling observed in these mutant mice. Furthermore, LDB2 was identified as an upregulated gene during the morphogenic stages of angiogenesis in a chick chorioallantoic membrane assay (25), and LDB2 expression in blood vessels in the lungs of mice was confirmed (25). There was no significant vascular phenotype in LDB2 mutant mouse embryos presumably because the homologue LDB1 may compensate for LDB2. However, more detailed investigations on blood vessel development in mutant mice and further elucidation of the role of LDB2 in pathological angiogenesis is required.

In conclusion, this study suggests that LDB2 plays a role in endothelial sprouting by regulating DLL4 expression via an oligomeric complex and that manipulation of the LDB2 complex could be exploited for the development of therapeutic strategies to inhibit aberrant angiogenesis.

\section{MATERIALS AND METHODS}

\section{Cell cultures}

Primary human umbilical vein endothelial cells (HUVEC) were purchased from Lonza Inc. and used between passage 4 and 8 . Endothelial cells were cultured in gelatin-coated plates in Medium 199 (Hyclone) containing 20\% FBS (Lonza), $3 \mathrm{ng} / \mathrm{ml}$ basic-FGF (R\&D System), 5 units/ml heparin (Sigma), and 1\% penicillin-streptomycin (Gibco).

\section{Tube formation assay and in vitro morphogenesis on 2-D Matrigel}

Tube formation was assayed as previously described (26). Briefly, $300 \mu$ lof Matrigel (BD Biosciences) was added to the wells of a 24-well plate and allowed to polymerize for $20 \mathrm{~min}$ at $37^{\circ} \mathrm{C}$. HUVECs were harvested, resuspended in Medium 199 , and seeded onto Matrigel $\left(0.8 \times 10^{5}\right.$ cells/well).

\section{Transfection of siRNA and DNA}

HUVECs of $60-80 \%$ confluence were transfected with control siRNA (Santa Cruz Biotech.; sc-37007) or LDB2 specific siRNA (Santa Cruz Biotech.; sc-38023) using Lipofectamine (Invitrogen). HUVECs were transfected with plasmid DNAs using Lipofectamine LTX and Plus reagent following manufacturer's instruction (Invitrogen). Total amount of DNA transfected was adjusted with the control vector plasmid. 


\section{Morpholino injection}

Micro-injections of morpholino oligonucleotides were carried out as previously described (26). Briefly, embryos were injected at the single-cell stage with 2-5 ng of control morpholino (Gene Tools). The sequences for the morpholino oligonucleotides used in this study are: $1 \mathrm{db} 2$ splicing morpholino: 5'-CGTACACACCTGAGAAAGCAGACAT-3'; ldb2 ATG morpholino: 5'-CATGTTGATTCCGTGTGCCGTTTGC-3'; mismatch control for Idb2 ATG morpholino; 5'-CATCTTCA TTGCGTCTGCCCTTTGC-3'.

\section{3-D Fibrin gel assay for endothelial sprouting}

Endothelial sprouting assays were performed as previously described (27) with some modifications. HUVECs were trypsinized and mixed with Cytodex microcarrier beads (Amersham Pharmacia) at a ratio of $10^{6}$ cells per 2,500 beads for $4 \mathrm{~h}$ at $37^{\circ} \mathrm{C}$. After incubation for $24 \mathrm{~h}$ at $37^{\circ} \mathrm{C}$, the coated beads were embedded into the fibrinogen with thrombin. The clots were overlaid with $2 \times 10^{4}$ human lung carcinoma fibroblasts (Wl-38) and incubated with $2 \mathrm{ml}$ of EGM-2 medium per well. Media was replaced every 2 days and vessel formation was analyzed after 3-5 days.

\section{qRT-PCR}

Total RNA was extracted and purified using the RNeasy kit (Qiagen). qRT-PCR was performed with $1 \times$ SYBR Green Mix (Invitrogen) with a Bio-Rad real-time PCR detection system. For each reaction, $100 \mathrm{ng}$ of total RNA was transcribed at $50^{\circ} \mathrm{C}$ for followed by a denaturing step at $95^{\circ} \mathrm{C}$ for $5 \mathrm{~min}, 40$ cycles of $95^{\circ} \mathrm{C}$ for $30 \mathrm{~s}$ and $60^{\circ} \mathrm{C}$ for $30 \mathrm{~s}$. Fluorescence data were collected and analyzed using an l-cycler (Bio-Rad). For normalization, human $\mathrm{GAPDH}$ was used as the endogenous control.

\section{Western blotting}

Cell pellets were lysed on ice with RIPA buffer supplemented with phosphatase inhibitor cocktail tablets (Roche) and then were mixed with $6 \times$ sample buffer. Protein samples were electrophoresed by SDS-PAGE and transferred to PVDF membranes. The membranes were probed with anti-LDB2 (1:500, Abcam), anti-DLL4 (1:500, Cell Signaling), and anti$\beta$-actin (1:1,000, Santa Cruz) antibodies and detection was performed with an ECL Plus detection kit (Amersham Bioscience).

\section{Luciferase assay}

The luciferase reporter assay was performed as described previously $(28,29)$. HUVECs that were plated onto a gelatincoated dish were transfected with different expression vectors along with reporter plasmids as described in the figures. The total amount of transfected plasmid DNA was adjusted with empty vector DNA. Luciferase activity was assayed $24 \mathrm{~h}$ after transfection. Cells were lysed with Passive lysis buffer (Promega), and luciferase activities in cell extracts were determined $24 \mathrm{~h}$ after transfection using a Dual luciferase assay system (Promega).

\section{CHIP assay}

The CHIP assay was performed using a CHIP kit (Millipore) as previously described, with some modification (30). Genomic DNA and protein were cross-linked by adding formaldehyde ( $1 \%$, final concentration) directly to the culture medium and incubating for $10 \mathrm{~min}$ at $37^{\circ} \mathrm{C}$. Then, the cells were harvested, lysed, and sonicated to generate 0.3-1.0 kb DNA fragments. After centrifugation, the cleared supernatant was incubated with anti-TAL1 (Santa Cruz), anti-GATA2 (Santa Cruz), anti-FLAG (Sigma), and $\operatorname{lgG}$ for immunoprecipitation. Co-immunoprecipitated and input DNAs were amplified by PCR, and amplified DNA was separated on a $1.5 \%$ agarose gel and visualized with ethidium bromide.

\section{Statistical analysis}

Statistical analysis was carried out using GraphPad Prism 4.0. Data are presented as mean \pm standard deviation (SD). Differences between groups were performed through an unpaired Student's $t$-test. A value of $\mathrm{P}<0.05$ was considered significant.

\section{ACKNOWLEDGEMENTS}

This research was supported by Basic Science Research Program through the National Research Foundation of Korea (NRF) funded by the Ministry of Education (2015R1D1A1A 01056703) and MSIP (2015R1A2A1A05001859), and by a grant of the Korea Healthcare technology R\&D Project, Ministry for Health \& Welfare Affairs (HI08C2149), Republic of Korea. This study was supported by a faculty research grant of Yonsei University College of Medicine for 2016 (201632-0063)

\section{CONFLICTS OF INTEREST}

The authors have no conflicting interests.

\section{REFERENCES}

1. Blanco R and Gerhardt H (2013) VEGF and Notch in tip and stalk cell selection. Cold Spring Harb Perspect Med 3, a006569

2. Gridley T (2010) Notch signaling in the vasculature. Curr Top Dev Biol 92, 277-309

3. Lobov IB, Renard RA, Papadopoulos N et al (2007) Delta-like ligand 4 (DII4) is induced by VEGF as a negative regulator of angiogenic sprouting. Proc Natl Acad Sci U S A 104, 3219-3224

4. Suchting S, Freitas C, le Noble F et al (2007) The Notch ligand Delta-like 4 negatively regulates endothelial tip cell formation and vessel branching. Proc Natl Acad Sci U S A $104,3225-3230$ 
5. Gerhardt $H$, Golding $M$, Fruttiger $M$ et al (2003) VEGF guides angiogenic sprouting utilizing endothelial tip cell filopodia. J Cell Biol 161,1163-1177

6. Hellstrom M, Phng LK, Hofmann JJ et al (2007) Dll4 signalling through Notch1 regulates formation of tip cells during angiogenesis. Nature 445, 776-780

7. Agulnick AD, Taira M, Breen JJ, Tanaka T, Dawid IB and Westphal H (1996) Interactions of the LIM-domainbinding factor Ldb1 with LIM homeodomain proteins. Nature 384, 270-272

8. Becker T, Ostendorff HP, Bossenz M et al (2002) Multiple functions of LIM domain-binding CLIM/NLI/Ldb cofactors during zebrafish development. Mech Dev 117, 75-85

9. Matthews JM and Visvader JE (2003) LIM-domain-binding protein 1: a multifunctional cofactor that interacts with diverse proteins. EMBO Rep 4, 1132-1137

10. Bach I, Carriere C, Ostendorff HP, Andersen B and Rosenfeld MG (1997) A family of LIM domain-associated cofactors confer transcriptional synergism between LIM and Otx homeodomain proteins. Genes Dev 11, 13701380

11. Wadman IA, Osada H, Grutz GG et al (1997) The LIM-only protein Lmo2 is a bridging molecule assembling an erythroid, DNA-binding complex which includes the TAL1, E47, GATA-1 and Ldb1/NLI proteins. EMBO J 16, 3145-3157

12. Song $\mathrm{SH}, \mathrm{Kim} A$, Ragoczy $T$, Bender MA, Groudine $M$ and Dean A (2010) Multiple functions of Ldb1 required for beta-globin activation during erythroid differentiation. Blood 116, 2356-2364

13. Bridge G, Monteiro R, Henderson S et al (2012) The microRNA-30 family targets DLL4 to modulate endothelial cell behavior during angiogenesis. Blood 120, 5063-5072

14. Mukhopadhyay M, Teufel A, Yamashita T et al (2003) Functional ablation of the mouse Ldb1 gene results in severe patterning defects during gastrulation. Development 130, 495-505

15. Corada M, Nyqvist D, Orsenigo F et al (2010) The Wnt/beta-catenin pathway modulates vascular remodeling and specification by upregulating Dll4/Notch signaling. Dev Cell 18, 938-949

16. Hayashi $\mathrm{H}$ and Kume $\mathrm{T}$ (2008) Foxc transcription factors directly regulate DII4 and Hey2 expression by interacting with the VEGF-Notch signaling pathways in endothelial cells. PLoS One 3, e2401

17. Seo S, Fujita H, Nakano A, Kang M, Duarte A and Kume T (2006) The forkhead transcription factors, Foxc1 and Foxc2, are required for arterial specification and lymphatic sprouting during vascular development. Dev Biol 294, 458-470

18. Wythe JD, Dang LT, Devine WP et al (2013) ETS factors regulate Vegf-dependent arterial specification. Dev Cell $26,45-58$

19. Sacilotto N, Monteiro R, Fritzsche M et al (2013) Analysis of DIl4 regulation reveals a combinatorial role for Sox and Notch in arterial development. Proc Natl Acad Sci U S A 110, 11893-11898

20. Patterson LJ, Gering M and Patient R (2005) Scl is required for dorsal aorta as well as blood formation in zebrafish embryos. Blood 105, 3502-3511

21. Visvader JE, Fujiwara Y and Orkin SH (1998) Unsuspected role for the T-cell leukemia protein SCL/tal-1 in vascular development. Genes Dev 12, 473-479

22. Lazrak M, Deleuze V, Noel D et al (2004) The bHLH $\mathrm{TAL}-1 / \mathrm{SCL}$ regulates endothelial cell migration and morphogenesis. J Cell Sci 117, 1161-1171

23. Deleuze V, Chalhoub E, El-Hajj R et al (2007) TAL-1/SCL and its partners $\mathrm{E} 47$ and $\mathrm{LMO} 2$ up-regulate $\mathrm{VE}$-cadherin expression in endothelial cells. Mol Cell Biol 27, 2687-2697

24. Yamada Y, Pannell R, Forster A and Rabbitts TH (2000) The oncogenic LIM-only transcription factor Lmo2 regulates angiogenesis but not vasculogenesis in mice. Proc Natl Acad Sci U S A 97, 320-324

25. Javerzat S, Franco M, Herbert J et al (2009) Correlating global gene regulation to angiogenesis in the developing chick extra-embryonic vascular system. PLoS One 4, e7856

26. Min JK, Cho YL, Choi JH et al (2007) Receptor activator of nuclear factor (NF)-kappaB ligand (RANKL) increases vascular permeability: impaired permeability and angiogenesis in eNOS-deficient mice. Blood 109, 1495-1502

27. Nakatsu MN and Hughes CC (2008) An optimized three-dimensional in vitro model for the analysis of angiogenesis. Methods Enzymol 443, 65-82

28. Choi HJ, Zhang H, Park H et al (2015) Yes-associated protein regulates endothelial cell contact-mediated expression of angiopoietin-2. Nat Commun 6, 6943

29. $\mathrm{Na} \mathrm{HH}$, Noh HJ, Cheong HM et al (2016) SETDB1 mediated FosB expression increases the cell proliferation rate during anticancer drug therapy. BMB Rep 49, 238-243

30. Song I, Jeong BC, Choi YJ et al (2016) GATA4 negatively regulates bone sialoprotein expression in osteoblasts. BMB Rep 49, 343-348 